\title{
Correlation Between Small and Dense Low Density Lipoprotein Cholesterol and Cardiovascular Events in Beijing Community Population
}

\section{Xiaona Wang}

Chinese PLA General Hospital

\section{Liang wang}

Chinese PLA General Hospital

\section{Ruihua Cao}

Chinese PLA General Hospital

\section{Xu Yang}

Chinese PLA General Hospital

\section{Wenkai Xiao}

Chinese PLA General Hospital

\section{Yun Zhang}

Chinese PLA General Hospital

\section{Ping Ye ( $\nabla$ yeping301@sina.com )}

Chinese PLA General Hospital https://orcid.org/0000-0003-4974-7087

\section{Research}

Keywords: sdLDL-C, major adverse cardiovascular events

Posted Date: November 19th, 2020

DOl: https://doi.org/10.21203/rs.3.rs-108427/v1

License: (c) (i) This work is licensed under a Creative Commons Attribution 4.0 International License. Read Full License 


\section{Abstract}

\section{Background}

The relationship between small dense low-density lipoprotein cholesterol (sdLDL-C) and different cardiovascular events has been observed in several large community studies, and the results have been controversial. However, there is currently no cross-sectional or longitudinal follow-up study on sdLDL-C in the Chinese hypertension population.

\section{Methods}

We analyzed the association of plasma sdLDL-C levels with major adverse cardiovascular events in 1325 subjects from a longitudinal follow-up community-based population in Beijing, China.

Results

During the follow-up period, a total of 191 subjects had MACEs. Cox regression analysis showed that sdLDL-C is a major risk factor for MACEs independent of sex, age, BMI, hypertension, diabetes, smoking, SBP, DBP, FBG, eGFR in the general community population $(1.013(1.001-1.025, \mathrm{P}<0.05))$, but the correlation disappeared after adjusting for TC and HDL-C in Model 3. Cox analysis showed that hypertension combined with high level of sdLDL-C was still the risk factor for MACEs ((2.079 (1.0394.148)).

Conclusions

Our findings in the Chinese cohort support that sdLDL-C is a risk factor for major adverse cardiovascular events in hypertension subjects.

\section{Background}

The incidence of coronary heart disease (CHD) is increasing; CHD affects the health and quality of life of patients and is even life-threatening. CHD is affected by a variety of risk factors, such as sex, age, family history, smoking history, hypertension, diabetes, dyslipidemia and so on [1]. Currently, it is recognized that dyslipidemia is the main target of CHD treatment; in particular, low-density lipoprotein cholesterol (LDL-C) plays an important role in the formation of atherosclerosis. The higher the level of LDL-C is, the greater the possibility of atherosclerosis is. At present, LDL is taken as the primary target for intervention. However, many patients with normal LDL-C levels will still develop cardiovascular disease [2,3]. Therefore, further studies on LDL subgroups have found that small dense low-density lipoprotein cholesterol (sdLDL-C) has a stronger atherogenic effect $[5,6]$. However, the data on the relationship between sdLDL-C and CHD worldwide are still limited. Therefore, the purpose of this study is to explore the relationship between sdLDL-C and major adverse cardiovascular events (MACEs) and its role in predicting MACEs.

\section{Methods}




\section{Subjects}

The research was conducted in the Pingguoyuan community in Beijing, China. A public recruitment announcement was first published on the community bulletin board with the consent of the community management committee. The subjects voluntarily participated in the study, which aimed to recruit community residents. Bedridden patients, or patients suffering from mental disorders or severe systemic diseases (including myocardial infarction, coronary artery disease, cerebrovascular events, severe liver insufficiency, severe renal insufficiency, immune system diseases, endocrine and metabolic disorders [except for Type 2 diabetes]) were excluded from this study. Initially, all the subjects received physical examinations at the community medical center. From September 2007 to January 2009, a total of 1828 subjects aged $\geq 18$ years were recruited. In the end, 1680 subjects were enrolled in this study, and the enrolled subjects were followed up for a second time between September 2017 and October 2018 via a face-to-face questionnaire by two cardiovascular physicians. An average follow-up time of 9.5 years (8.710.5 years) was completed by 1325 subjects, during which 191 major adverse cardiovascular events (MACEs) occurred, and 355 subjects were lost to follow-up. The proportion of subjects lost to follow-up was approximately $21.1 \%$, and the follow-up rate was $78.9 \%$. The entire protocol of this study was approved by the ethics committee of the PLA General Hospital, and each subject signed an informed consent form.

\section{Clinical data collection}

Medical history was collected by cardiovascular specialists with unified training. All the subjects were investigated to determine the following: cardiovascular history, hypertension history, diabetes history, family history, smoking history, drinking history, and medication history. A standardized questionnaire was used to investigate the subjects' name, sex, nationality, age, education level, occupation, marital status, family address, contact information and other information. Blood pressure, height, weight, and waist and hip circumference were measured by trained doctors. When measuring the height and weight, shoes, socks and caps were removed, and the height measurements were accurate to $1 \mathrm{~cm}$, and the weight meter measurements were accurate to $0.1 \mathrm{~kg}$. To measure the waist and hip circumference, any coat was removed and belts were loosened, and the tape measure was placed between the upper part of the hip bone and the lower part of the chest. The measurements were made while the subject was exhaling and their abdomen was relaxed, and the readings were accurate to $1 \mathrm{~cm}$. Prior to measuring their blood pressure, the subjects rested in a sitting position for 5 minutes. Then, blood pressure was measured twice with an interval of 1-2 minutes, and the mean value was calculated.

Blood was drawn from subjects with an empty stomach by specially trained nurses. Patients who had not been diagnosed with diabetes mellitus were examined by an oral glucose tolerance test (OGTT). Fasting blood glucose and postprandial blood glucose, serum TC, TG, HDL-C, LDL-C and uric acid (UA) were measured with a Roche Diagnostics GmbH kit (Mannheim, Germany) using a Roche 6000 automatic biochemical analyzer (Roche 6000). Serum creatinine (CR) was determined by a Roche enzyme assay kit on the Hitachi 7600 automatic analyzer (Hitachi, Tokyo, Japan). The sdLDL "Seiken" kit (Denka Seiken 
Co. Ltd, Tokyo, Japan) was used to detect the plasma sdLDL levels on the Hitachi 7180 automatic biochemical analyzer (Hitachi, Japan) [6]. According to the standards of the WHO lipid reference laboratory, all the blood samples were analyzed in the same laboratory.

\section{Definition of variables}

Smoking is defined as smoking at least 1 cigarette a day for at least 1 year. Exercise is defined as at least 1 hour of each exercise performed 3 to 5 times a week. Hypertension is defined as: mean systolic blood pressure (SBP) $\geq 140 \mathrm{mmHg}$ and/or mean diastolic blood pressure (DBP) $\geq 90 \mathrm{mmHg}$, and/or regular use of antihypertensive drugs. Diabetes is defined as: intravenous fasting blood glucose level $\geq 7.1 \mathrm{mmol} / \mathrm{L}$, and/or 2 hours after eating blood glucose level $\geq 11.1 \mathrm{mmol} / \mathrm{L}$, or use of hypoglycemic drugs or insulin. $\mathrm{BMI}=$ weight $(\mathrm{kg}) /$ height $^{2}\left(\mathrm{~m}^{2}\right)$; Non-HDL-C=TC $(\mathrm{mmol} / \mathrm{L})-\mathrm{HDL}-\mathrm{C}(\mathrm{mmol} / \mathrm{L}) ; \mathrm{eGFR}=141 \times \mathrm{min}(\mathrm{Scr} / \mathrm{k}, 1)^{\mathrm{a}} \times$ $\max (\mathrm{Scr} / \mathrm{K}, 1)^{-1.209} \times 0.993^{\mathrm{Age}} \times 1.018$ [female] (or $\times 1.159$ [male]); Scr is blood creatinine $(\mathrm{mg} / \mathrm{dL}) ; \mathrm{K}$ Values are: 0.7 for women and 0.9 for men; $\alpha$ is -0.329 for women and -0.411 for men.

The definition of MACE comprised nonfatal myocardial infarction, newly diagnosed CHD (identified by coronary artery imaging or receiving coronary revascularization), stroke (ischemic or hemorrhagic) and cardiovascular mortality.

\section{Statistical analyses}

The categorical variables are expressed as numbers and percentages, and the continuous variables are expressed as the mean \pm standard deviation or median (quartile). $T$ test was used for normally distributed data, while the nonparametric Mann-Whitney U test was used for nonnormally distributed data, and the chi-square test used for classified data. For the comparison between groups, analysis of variance was used when the data were homogeneous, and Kruskal-Wallis test was used for the comparison between groups when the data were not normally distributed or the variance was not uniform. The quartiles of the sdLDL-C levels were labeled as Q1, Q2, Q3 and Q4. The subjects were divided into four groups according to whether or not they had hypertension: $\mathrm{H} 1$ : high levels of sdLDL-C and hypertension; $\mathrm{H} 2$ : high levels of sdLDL-C and nonhypertensive; H3: hypertensive and low levels of sdLDL-C; H4: low levels of sdLDL-C and nonhypertensive. Spearman correlation analysis and multiple linear regression analysis were used to evaluate the correlation between the plasma sdLDL-C levels and biomarkers. The relationship between the sdLDL-C levels and MACEs was analyzed by Cox proportional hazard regression model. SPSS for Windows version 20.0 (SPSS, Chicago, IL, USA) was used for statistical analysis, $\mathrm{P}<0.05$ was considered statistically significant.

\section{Results}

\section{Baseline characteristics of the study population}

This study initially enrolled 1680 subjects, with an average follow-up time of 9.5 years (8.7-10.5 years). For various reasons, 355 subjects were lost to follow-up. A successful follow-up rate of $78.9 \%$ (1325 
subjects) was achieved. One hundred ninety-one cases of MACEs occurred. In the end, the data of 1325 subjects were used in the analysis of this study, including the data of 642 males (48.5\%) and 683 females (51.5\%), and these subjects had an average age of $58.99 \pm 11.28$ years. A stratified analysis was made according to whether MACEs occurred and the baseline characteristics and laboratory data of the study population were statistically analyzed as shown in Table 1. There were differences in age, sex, smoking, diabetes, SBP, HDL-C, LDL-C, eGFR and sdLDL-C between the patients with MACEs and those without MACEs $(P<0.05)$. The main characteristics of the population with MACEs were: advanced age, male, smoking, diabetes, higher levels of SBP, LDL-C and sdLDL-C, and lower levels of HDL-C and eGFR.

\section{Correlation analysis of baseline sdLDL-C and other indicators}

In the univariate model, the baseline sdLDL-C level was positively correlated with age, BMI, SBP, DBP, TC, $T G, L D L, F B G$, and UA $(P<0.05)$. Multiple linear regression analysis showed that $T G, L D L-C$, and circulating sdLDL-C levels were positively correlated $(P<0.05)($ Table 2$)$.

\section{Correlation analysis of sdLDL-C and major adverse cardiovascular events}

During the follow-up period, a total of 191 subjects had MACEs. Cox regression analysis showed that sdLDL-C was the main risk factor for MACEs (Table 3). The hazard ratio of the highest quartile $(>52.2$ $\mathrm{ng} / \mathrm{ml}$ ) to the lowest quartile $(\leq 25.18 \mathrm{ng} / \mathrm{ml}$ ) of sdLDL-C was 1.008 , and the $95 \% \mathrm{Cl}$ was $1.004-1.019$ (P $<0.05)$. Model 1 , which substituted sex and age into the equation, derived a hazard ratio between the highest quartile and the lowest quartile of sdLDL-C that was 1.014, and the $95 \% \mathrm{Cl}$ was $1.003-1.026$ ( $\mathrm{P}$ $<0.05$ ). Model 2 substitutes traditional cardiovascular risk factors into the equation, and the results show that sdLDL-C is a major risk factor for MACEs independent of sex, age, BMI, hypertension, diabetes, smoking, SBP, DBP, FBG, eGFR in the general community population $(1.013(1.001-1.025, P<0.05))$, but the correlation disappeared after adjusting for TC and HDL-C in Model 3.

\section{Correlation analysis of sdLDL-C and major adverse cardiovascular events in hypertensive population}

Cox analysis was performed based on whether the subjects were hypertensive (Table 4). The results showed that when other risk factors were not adjusted, hypertension and high levels of sdLDL-C were risk factors for MACEs in the elderly population (2.604 (1.369-4.953, $\mathrm{P}<0.01)$ ). Model 1, adjusted for sex and age, showed that sdLDL-C is a risk factor for MACEs $(2.539$ (1.333-4.835, $\mathrm{P}<0.01))$. Model 3 , adjusted for sex, age, BMI, diabetes, smoking, SBP, DBP, TC, HDL-C, FBG, eGFR, showed that hypertension combined with high level of sdLDL-C was still the risk factor for MACEs ((2.079 (1.039-4.148)). The data suggest that sdLDL-C is an independent risk factor for major adverse cardiovascular events in people with hypertension (Figure 1).

\section{Discussion}

LDL-C plays an important role in the occurrence and outcome of atherosclerosis and cardiovascular diseases. Reasonable application of statins can play a positive role in preventing cardiovascular 
events[7,8], but there are also many clinical studies that have found that the risk of cardiovascular disease (CVD) is reduced to less than $30 \%$ after the application of statin lipid-lowering drugs. There may be other important risk factors to be considered [9-11]. The occurrence and development of atherosclerosis (AS) is not only related to the total amount of cholesterol, but may also be closely related to its heterogeneity [12]. According to the size and density, LDL can be divided into different subtypes, including large and light LDL (large buoyant low-density lipoprotein, IbLDL), intermediate-density LDL (intermediate-density lipoprotein, IDL), and small and dense LDL (small dense low-density lipoprotein, sdLDL). Many scholars believe that sdLDL has a stronger atherogenic effect than other LDL subtypes, as shown by different methodological studies, and sdLDL levels may have more predictive value for cardiovascular disease than LDL-C.

Whether sdLDL-C is a risk factor for CAD independent of other traditional lipid indicators is still controversial. A case-control study [13] enrolled 109 nonfatal myocardial infarction patients and 121 control patients and found that sdLDL-C was independently associated with the risk of myocardial infarction even after adjusting for risk factors such as age, sex, and weight. In a Japanese prospective study [14], 2034 patients without cardiovascular disease were followed for an average 11.7 years. The results showed that an increased level of sdLDL-C was associated with an increased risk of CVD. For every $10 \mathrm{mg} / \mathrm{dL}$ increase in sdLDL-C, the risk of CVD increased by 1.21 (95\% Cl: 1.12-1.31). Other studies found that sdLDL-C is a risk factor for CAD in people with diabetes [15], kidney disease [16] and liver transplants [17]. Other research has shown that sdLDL-C has a stronger relationship with cIMT progression than LDL-C[18]. The ARIC study [19] included a total of 11,419 patients followed for 11 years and found that sdLDL-C is a risk factor for coronary heart disease, but after adjusting for LDL-C, apo B and TC, the predictive value of sdLDL-C is weakened or disappears. Some scholars [20] believe that in patients after myocardial infarction, sdLDL is associated with a reduced risk of all-cause death and noncardiovascular death. In this study, we found that after adjusting for confounding factors (sex, age, BMI, hypertension, diabetes, smoking, TC, HDL-C, FBG, eGFR), the circulating sdLDL-C level was not an independent risk factor for MACEs. To further explore the relationship between sdLDL-C and MACEs in patients with hypertension, it was observed that sdLDL-C was an independent risk factor for MACE (2.079 $(1.039-4.148, \mathrm{P}<0.05))$.

LDL-C is the main lipid risk factor for the development of AS, and sdLDL-C is considered to be the main LDL-C component that causes AS. Very low-density lipoprotein (VLDL), which is rich in triglycerides secreted by the liver, is defatted by lipoprotein lipase, and the remaining triglycerides in VLDL are transferred to LDL particles through cholesterol ester transfer protein. sdLDL is the product of LDL particles defatted by liver lipase $[21,22]$. The half-life of sdLDL is longer than that of IbLDL $(3.10$ days vs. 1.95 days) [23], and it is more prone to oxidation reactions. Therefore, sdLDL is more effective in causing atherosclerosis than LDL-C, especially in people with metabolic syndrome, obesity and diabetes [24-28]. sdLDL-C can participate in atherosclerosis through a variety of mechanisms. Because of its smaller particles, sdLDL-C is more likely to penetrate the endothelial barrier and migrate to the blood vessel wall and is more likely to be oxidized. The decrease in clearance rate increases the possibility of chemical modification in plasma $[29,30]$, which is considered to facilitate progression of the atherosclerotic plaque. 
sdLDL-C can also participate in inflammation by activating immune cells and participate in the development of coronary heart disease [31]. In addition, sdLDL-C is related to the presence of macrophages in plaques, which may be related to unstable arterial plaques [32].

Increasing numbers of studies have confirmed the value of sdLDL-C in CVD risk prediction. SdLDL-C has been listed as one of the newly discovered important cardiovascular risk factors in the adult treatment group of the National Cholesterol Education Program (NCEP) [33]. Apparently, cholesterol- and LDLlowering compounds are also effective in the reduction of sdLDL levels. In addition, improving the lipid profile, especially reducing triglyceride levels, adopting an appropriate regimen, and changing one's lifestyle, can decrease sdLDL levels[34]. In this study, we reported a direct relationship between plasma sdLDL levels and MACEs in a large sample community population. However, since this is a communitybased study, it is limited by race, ethnicity and geographical location.

\section{Abbreviations}

sdLDL-C: small dense low density lipoprotein cholesterol; SBP: Systolic blood pressures; DBP: diastolic blood pressures; FBG: fasting blood glucose; TC: total cholesterol; TGs: triglycerides; LDL-C:low density lipoprotein cholesterol; HDL-C: high density lipoprotein cholesterol; MACE: major adverse cardiovascular events

\section{Declarations}

Ethics approval and consent to participate: The study was approved by the ethics committee of the People's Liberation Army General Hospital, and each subject provided informed written consent.

Consent for publication: Not applicable

Availability of data and materials: Not applicable

Competing interests: The authors declare that they have no competing interests

Funding: This research is supported by the the Key Science and Technology Foundation of China (2020YFC2004805) to Dr. Sheng Li.

Authors' contributions: $\mathrm{XW}$ and $\mathrm{PY}$ designed the study; RC, $\mathrm{XY}, \mathrm{WX}$ and $\mathrm{YZ}$ participated in acquisition of data; $X W, P Y$ and $Y B$ researched and evaluated the literature; $X W$ undertook the statistical analysis and wrote the first draft of the manuscript. All authors read and approved the final manuscript

Acknowledgements: Acknowledgments and disclosures: We thank colleagues at the Department of Laboratory Medicine, the PLA General Hospital for help with biochemical measurements. We are also grateful to all study participants for their participation in the study

\section{References}


1. Ueda Y, Ogasawara N, Matsuo K, et al. Acute coronary syndrome: insight from angioscopy[J]. Circ J, 2010, 74(3):411-417.

2. Goel PK, Ashfaq F, Khanna R, et al. The Association Between Small Dense Low Density Lipoprotein and Coronary Artery Disease in North Indian Patients[J]. Indian J Clin Biochem, 2017, 32(2): 186-192.

3. Cholesterol Treatment Trialists' (CTT) Collaboration, C Baigent, L Blackwell, et al. Efficacy and safety of more intensive lowering of LDL cholesterol: a meta-analysis of data from 170, 000 participants in 26 randomised trials[J]. Lancet, 2010, 376(9753):1670-1681.

4. J V Patel, M J Caslake, A Vyas, et al. Triglycerides and small dense low density lipoprotein in the discrimination of coronary heart disease risk in South Asian populations[J]. Atherosclerosis, 2010, 209(2):579- 584.

5. SakaiK, KobaS, Nakamura Y, et al.Small dense low-density lipoprotein cholesterol is a promising biomarker for secondary prevention in older men with stable coronary artery disease[J]. Geriatr Gerontol Int, 2018, 18(6):965- 972.

6. Li, J-J, Inflammation in coronary artery diseases[J]. Chinese medical journal, 2011. 124(21): p 35683575.

7. de Vries FM, Denig P, Pouwels KB, et al. Primary prevention of major cardiovascular and cerebrovascular events with statins in diabetic patients:a meta-analysis[J]. Drugs,2012,72(18):23652373区

8. Chen Y H, Feng B, Chen Z W. Statins for primary prevention of cardiovascular and cerebrovascular events in diabetic patients without established cardiovascular diseases: a meta-analysis[J]. Exp Clin Endocrinol Diabetes, 2012,120(2):116-120区

9. Hirayama S, Miida T. Small dense LDL: An emerging risk factor for cardiovascular disease[J]. Clin Chim Acta, 2012, 414: 215-224区

10. Austin MA, Breslow JL, Hennekens $\mathrm{CH}$, et al. Low-density lipoprotein subclass patterns and risk of myocardial infarction[J]. JAMA, 1988,260(13): 1917-1921.

11. AraiH, Kokubo Y, Watanabe M, et al. Small dense low-density lipoproteins cholesterol can predict incident cardiovascular disease in an urban Japanese cohort:the Suita study[J]. J Atheroscler Thromb, 2013, 20(2):195-203.

12. JinJL, ZhangHW, Cao YX, et al. Association of small dense low-density lipoprotein with cardiovascular outcome in patients with coronary artery disease and diabetes: a prospective, observational cohort study[J]. Cardiovasc Diabetol, 2020 Apr 3;19(1):45.

13. Shen $H, X u$ Y, Lu J, et al. Small dense low-density lipoprotein cholesterol was associated with future cardiovascular events in chronic kidney disease patients[J]. BMC Nephrol 17, 143 (2016).

14. SiddiquiMB, ArshadT, Patel S, et al. Small Dense Low-Density Lipoprotein Cholesterol Predicts Cardiovascular Events in Liver Transplant Recipients[J]. Hepatology, 2019 Jul;70(1):98-107.

15. HoogeveenRC, Gaubatz JW, Sun WS, et al. Small dense low-density lipoprotein-cholesterol concentrations predict risk for coronary heart disease: the Atherosclerosis Risk In Communities (ARIC) study[J]. Arterioscler Thromb Vasc Biol, 2014, 34(5):1069-1077. 
16. PokharelY, TangY, Bhardwaj B, et al. Association of low-density lipoprotein pattern with mortality after myocardial infarction: Insights from the TRIUMPH study[J]. J Clin Lipidol, 2017, 11(6):14581470.

17. C Baigent, A Keech, P M Kearney, et al. Efficacy and safety of cholesterol-lowering treatment: prospective meta-analysis of data from 90,056 participants in 14 randomised trials of statins[J]. Lancet, 2005, 366(9493):1267-1278.

18. Ikezaki H, Furusyo N, Yokota Y, et al. Small Dense Low-Density Lipoprotein Cholesterol and Carotid Intimal Medial Thickness Progression. J Atheroscler Thromb, 2020 Oct 1;27(10):1108-1122.

19. Afilalo J, Majdan A A, Eisenberg M J. Intensive statin therapy in acute coronary syndromes and stable coronary heart disease: a comparative meta-analysis of randomised controlled trials[J]. Heart, 2007,93(8):914-921.

20. Packard C J. Small dense low-density lipoprotein and its role as all independent predictor of cardiovascular disease[J]. Curr Opin Lipidol, 2006, 17(4):412-417.

21. Hirayama, S. and T. Miida, Small dense LDL: An emerging risk factor for cardiovascular disease[J]. Clinica chimica acta; international journal of clinical chemistry, 2012. 414: p. 215-224.

22. J D Otvos, E J Jeyarajah, D W Bennett, et al. Development of a proton nuclear magnetic resonance spectroscopic method for determining plasma lipoprotein concentrations and subspecies distributions from a single, rapid measurement[J]. Clinical chemistry, 1992. 38(9): p. 1632-1638.

23. ThongtangN, DiffenderferMR, Esther M M Ooi , et al. Metabolism and proteomics of large and small dense LDL in combined hyperlipidemia: effects of rosuvastatin[J]. Journal of lipid research, 2017. 58(7): p. 1315-1324.

24. M A Austin, H Horowitz, E Wijsman, et al. Bimodality of plasma apolipoprotein B levels in familial combined hyperlipidemia[J]. Atherosclerosis, 1992. 92(1): p. 67-77.

25. AyyobiA F, McGladdery S H, McNeely M J, et al. Small, dense LDL and elevated apolipoprotein B are the common characteristics for the three major lipid phenotypes of familial combined hyperlipidemia[J]. Arteriosclerosis, thrombosis, and vascular biology, 2003. 23(7): p. 1289-1294.

26. Berneis K, Jeanneret C, Muser J, et al. Low-density lipoprotein size and subclasses are markers of clinically apparent and non-apparent atherosclerosis in type 2 diabetes[J]. Metabolism: clinical and experimental, 2005. 54(2): p. 227-234.

27. R Goldberg, M Temprosa, J Otvos, et al. Lifestyle and metformin treatment favorably influence lipoprotein subfraction distribution in the Diabetes Prevention Program[J]. The Journal of clinical endocrinology and metabolism, 2013. 98(10): p. 3989-3998.

28. NikolicD, Katsiki N, Montalto G, et al. Lipoprotein subfractions in metabolic syndrome and obesity: clinical significance and therapeutic approaches[J]. Nutrients, 2013. 5(3): p. 928-948.

29. Steinberg D, Parthasarathy S, Carew TE, et al. Beyond cholestero1. Modifications of low-density lipoprotein that increase its atherogenicity [J]. N Engl J Med, 1989,320(14):915-924.

30. aakkola O, Solakivi T, Tertov VV, et al. Characteristics of low-density lipoprotein subfractions from patients with coronary artery disease[J]. Coron Artery Dis, 1993, 4(4):379-385. 
31. Izumida T, Nakamura Y, Hino Y, et al. Combined Effect of Small Dense Low-Density Lipoprotein Cholesterol (sdLDL-C) and Remnant-Like Particle Cholesterol (RLP-C) on Low-Grade Inflammation[J]. J Atheroscler Thromb, 2020 Apr 1;27(4):319-330.

32. ZambonA, Puato $M$, Faggin $E$, et al. Lipoprotein remnants and dense LDL are associated with features of unstable carotid plaque: a flag for non-HDL-C [J]. Atherosclerosis, 2013, 230(1): 106-109.

33. Third Report of the National Cholesterol Education Program (NCEP) Expert Panel on Detection, Evaluation and Treatment of High Biood Cholesterol in Adults(Adult Tratment Panel III) final report[J]. Circulation, 2002, 106(25):3143 -3421.

34. Alizadeh-Fanalou S, Nazarizadeh A, Alian F, et al. Small dense low-density lipoprotein-lowering agents. Biol Chem, 2020 Sep 25;401(10):1101-1121.

\section{Tables}

\section{Table1. Baseline characteristics and laboratory test results of subjects}




\begin{tabular}{|c|c|c|c|c|}
\hline & All subjects & Mace & Non-mace[ & \\
\hline & $\bigotimes n=1325 \rrbracket$ & $\bigotimes n=1134 \rrbracket$ & $\bigotimes n=191 \rrbracket$ & \\
\hline age『प्रा & $58.99 \pm 11.28$ & $57.95 \pm 11.15$ & $65.90 \pm 9.57$ & $\llbracket 0.001$ \\
\hline male(n (\%)) & $642 \rrbracket 48.5 \rrbracket$ & $508 \otimes 44.8 \rrbracket$ & 102邓53.4】 & $\otimes 0.001$ \\
\hline 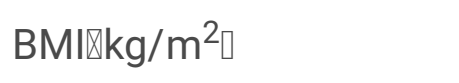 & $25.48 \pm 3.44$ & $25.44 \pm 3.36$ & $25.70 \pm 3.95$ & 0.353 \\
\hline smoking(n (\%)) & $359 \llbracket 27.1 \rrbracket$ & $286 \llbracket 25.2 \rrbracket$ & $62 \rrbracket 32.5 \rrbracket$ & $₫ 0.001$ \\
\hline diabetes(n (\%)) & $232 \bigotimes 17.5 \rrbracket$ & 177ه15.6ه & $44 \llbracket 23.0 \rrbracket$ & $\varangle 0.001$ \\
\hline SBP $\triangle \mathrm{mmHg} \rrbracket$ & $119.63 \pm 17.55$ & $118.62 \pm 17.33$ & $126.46 \pm 17.55$ & $\varangle 0.001$ \\
\hline $\mathrm{DBP} \triangle \mathrm{mmHg} \rrbracket$ & $76.55 \pm 11.22$ & $76.65 \pm 11.27$ & $75.85 \pm 10.87$ & 0.389 \\
\hline TC邓mmol/L】 & $5.02 \pm 0.93$ & $5.01 \pm 0.92$ & $5.0769 \pm 0.95$ & 0.424 \\
\hline TG \mmol/L】 & $1.5 \rrbracket 1.1 \rrbracket 2.1 \rrbracket$ & $1.5 \rrbracket 1.1 \rrbracket 2.11 \rrbracket$ & $1.6 \rrbracket 1.2 \rrbracket 2.4 \rrbracket$ & 0.228 \\
\hline HDL-C邓mmol/L】 & $1.37 \pm 0.37$ & $1.39 \pm 0.35$ & $1.25 \pm 0.43$ & $\nabla 0.001$ \\
\hline LDL-C $₫ \mathrm{mmol} / \mathrm{L} \otimes$ & $2.92 \pm 0.72$ & $2.89 \pm 0.71$ & $3.09 \pm 0.73$ & 0.001 \\
\hline FBG囚mmol/L】 & $5.38 \pm 1.66$ & $5.34 \pm 1.62$ & $5.63 \pm 1.87$ & 0.067 \\
\hline $\operatorname{eGFR}\left(\mathrm{ml} / \mathrm{min} / 1.73 \mathrm{~m}^{2}\right)$ & $94.17 \pm 14.35$ & $95.11 \pm 13.91$ & $87.10 \pm 15.63$ & $\varangle 0.001$ \\
\hline UA $₫ \mathrm{mmol} / \mathrm{L} \rrbracket$ & $293.87 \pm 76.59$ & $292.40 \pm 75.55$ & $304.08 \pm 82.99$ & 0.066 \\
\hline sdLDL-C(ng/ml) & $40.25 \pm 19.55$ & $42.89 \pm 20.43$ & $40.16 \pm 19.45$ & 0.035 \\
\hline $\begin{array}{l}\text { TC, total cholesterol; HD } \\
\text { lipoprotein cholesterol; } \\
\text { index; FBG, fast blood gl } \\
\text { dense low density lipopr }\end{array}$ & $\begin{array}{l}\text { gh-density lipop } \\
\text { stolic blood pres } \\
\text { eGFR, estimate } \\
\text { holesterol }\end{array}$ & $\begin{array}{l}\text { cholesterol; TC } \\
\text { DBP, diastolic } \\
\text { merular filtratio }\end{array}$ & $\begin{array}{l}\text { yceride; LDL-C, } \\
\text { pressure; BMI, b } \\
\text { 囚A, uric acid; } s\end{array}$ & $\begin{array}{l}\text { density } \\
\text { mass } \\
\text { L-C, small }\end{array}$ \\
\hline
\end{tabular}

\section{Table $2 \square$ Clinical factors affecting sdLDL-C}




\begin{tabular}{|c|c|c|c|c|}
\hline & \multicolumn{4}{|c|}{ Baseline sdLDL-C(ng/ml) } \\
\hline & $r$ & $P$ & $\beta$ & $P$ \\
\hline 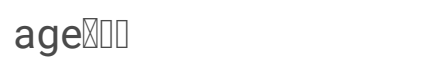 & 0.118 & $₫ 0.001$ & 0.001 & 0.992 \\
\hline male $\% \rrbracket$ & -0.027 & 0.409 & -4.346 & 0.095 \\
\hline 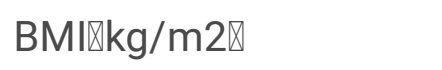 & 0.113 & 0.001 & 0.048 & 0.637 \\
\hline smoking $\otimes \% \square$ & -0.017 & 0.613 & -0.181 & 0.839 \\
\hline diabetes $₫ \% \bigotimes$ & 0.057 & 0.081 & 2.096 & 0.203 \\
\hline $\mathrm{SBP} \otimes \mathrm{mmHg} \rrbracket$ & 0.073 & 0.026 & 0.003 & 0.054 \\
\hline $\mathrm{DBP} \rrbracket \mathrm{mmHg} \mathbb{}$ & 0.141 & $₫ 0.001$ & 0.007 & 0.874 \\
\hline $\mathrm{TC} \triangle \mathrm{mmol} / \mathrm{L} \mathbb{}$ & 0.533 & $₫ 0.001$ & 0.244 & 0.862 \\
\hline TG $₫ \mathrm{mmol} / \mathrm{L} \rrbracket$ & 0.432 & $₫ 0.001$ & 5.821 & $\nabla 0.001$ \\
\hline $\mathrm{HDL}-\mathrm{C} \rrbracket \mathrm{mmol} / \mathrm{L} \rrbracket$ & -0.206 & $\otimes 0.001$ & -2.668 & 0.155 \\
\hline LDL-C $\otimes m m o l / L \otimes$ & 0.535 & $\llbracket 0.001$ & 12.976 & $\nabla 0.001$ \\
\hline FBG $₫ \mathrm{mmol} / \mathrm{L} \rrbracket$ & 0.112 & 0.001 & 0.152 & 0.712 \\
\hline $\mathrm{eGFR}\left(\mathrm{ml} / \mathrm{min} / 1.73 \mathrm{~m}^{2}\right)$ & 0.062 & 0.060 & 2.798 & 0.102 \\
\hline UADmmol/L】 & 0.146 & $\varangle 0.001$ & 0.012 & 0.151 \\
\hline
\end{tabular}

TC, total cholesterol; HDL-C, high- density lipoprotein cholesterol; TG, triglyceride; LDL-C, low-density lipoprotein cholesterol; SBP, systolic blood pressure; DBP, diastolic blood pressure; BMI, body mass index; FBG, fast blood glucose; eGFR, estimated glomerular filtration rate囚UA, uric acid; sdLDL-C, small dense low density lipoprotein cholesterol

Table 3 Cox proportional hazards models analysis for associations between baseline sdLDL-C levels and MACE

\begin{tabular}{|lllll|}
\hline \multicolumn{2}{|l}{ HR $(\mathbf{9 5 \%}$ Cl) } & Model1 & Model2 & \multicolumn{1}{l|}{ Model3 } \\
\hline Q1 & 1 & 1 & 1 & 1 \\
\hline Q2 & $1.005(0.962-1.049)$ & $1.009(0.967-1.953)$ & $1.007(0.964-1.052)$ & $1.007(0.958-1.059)$ \\
\hline Q3 & $1.008(0.986-1.031)$ & $1.018(0.994-1.042)$ & $1.018(0.994-1.043)$ & $1.022(0.993-1.052)$ \\
\hline Q4 & $1.008(1.004-1.019) *$ & $1.014(1.003-1.026)^{*}$ & $1.013(1.001-1.025) *$ & $1.005(0.989-1.021)$ \\
\hline
\end{tabular}


Models are defined as follows: model 1=adjusted for age and gender; model 2=adjusted for model $1+$ presence of BMI, hypertension, diabetes, smoking, SBP, DBP, FBG, eGFR; model 3=adjusted for model $2+$ TC\HDL-C

MACE, major adverse cardiovascular event.

$* P<0.05$

Table 4 Cox proportional hazards models analysis for associations between baseline sdLDL-C levels and MACE in hypertension

\begin{tabular}{|c|c|c|c|c|}
\hline \multicolumn{5}{|c|}{$\mathrm{HR}(95 \% \mathrm{Cl})$} \\
\hline & Unjust & Model1 & Model2 & Model3 \\
\hline $\mathrm{H} 1$ & 1 & 1 & 1 & 1 \\
\hline $\mathrm{H} 2$ & $1.117(0.470-2.653)$ & $1.288(0.543-3.056)$ & $1.251(0.521-3.004)$ & $1.361(0.515-3.596)$ \\
\hline H3 & $1.799(0.911-3.553)$ & $2.003(1.007-3.985)^{\star}$ & $1.898(0.953-3.778)$ & $2.601(1.122-6.027)^{\star}$ \\
\hline H4 & $\begin{array}{l}2.604(1.369- \\
4.953)^{\star \star}\end{array}$ & $\begin{array}{l}2.539(1.333- \\
4.835)^{\star \star}\end{array}$ & $\begin{array}{l}2.734(1.423- \\
5.250)^{\star \star}\end{array}$ & $2.079(1.039-4.148)^{\star}$ \\
\hline
\end{tabular}

Models are defined as follows: model 1=adjusted for age and gender; model 2=adjusted for model $1+$ presence of BMI, diabetes, smoking, SBP, DBP, FBG, eGFR; model 3=adjusted for model 2 + TC $\triangle \mathrm{HDL}-\mathrm{C}$ MACE, major adverse cardiovascular event.

** $\mathrm{P}<0.01 ; * \mathrm{P}<0.05$

\section{Figures}




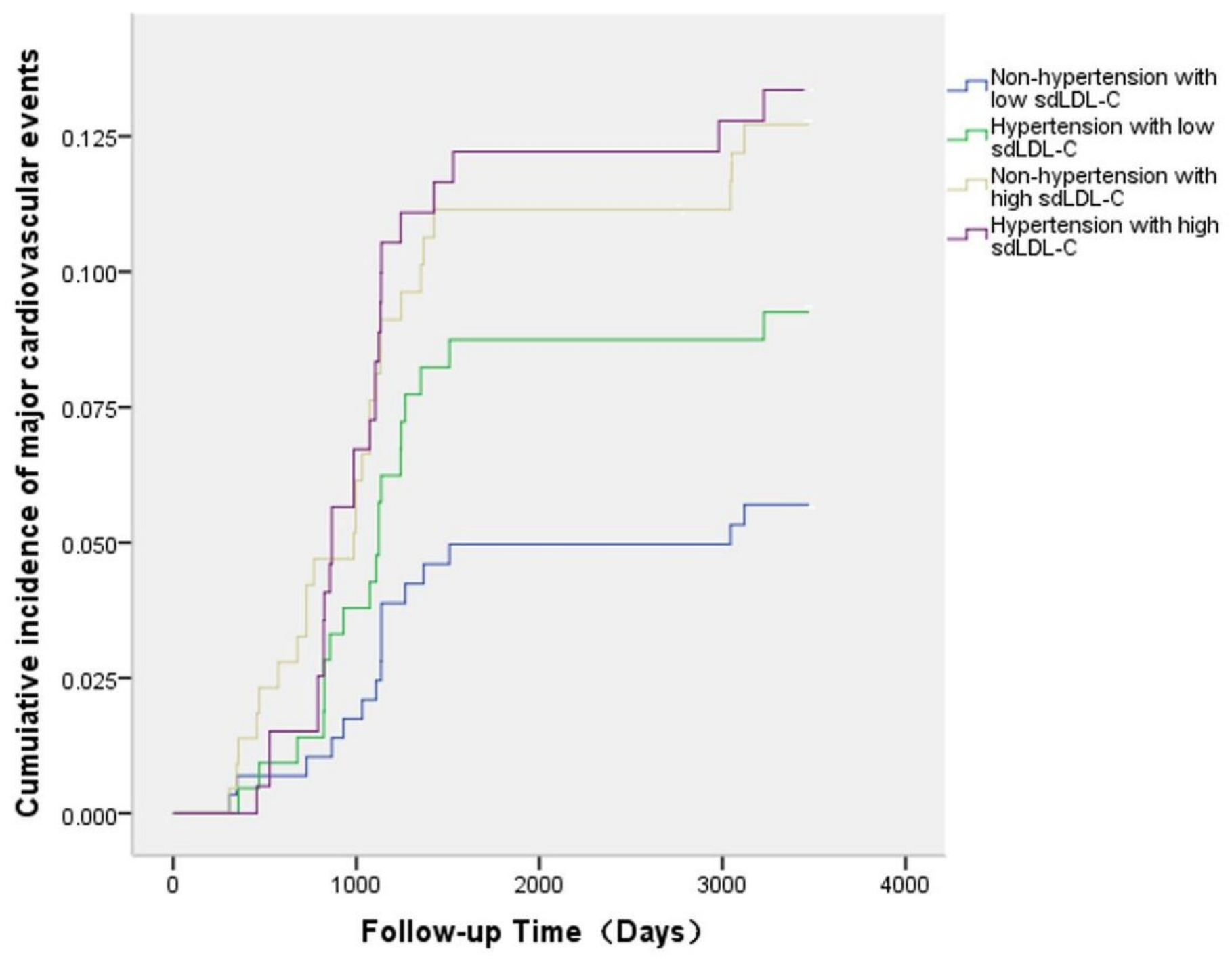

Figure 1

Risk for cardiovascular events by baseline sdLDL-C level. Kaplan-Meier survival curves indicating cumulative incidence of major adverse cardiovascular events across baseline sdLDL-C categories. Groups are indicated by colours; $p<0.001$ 


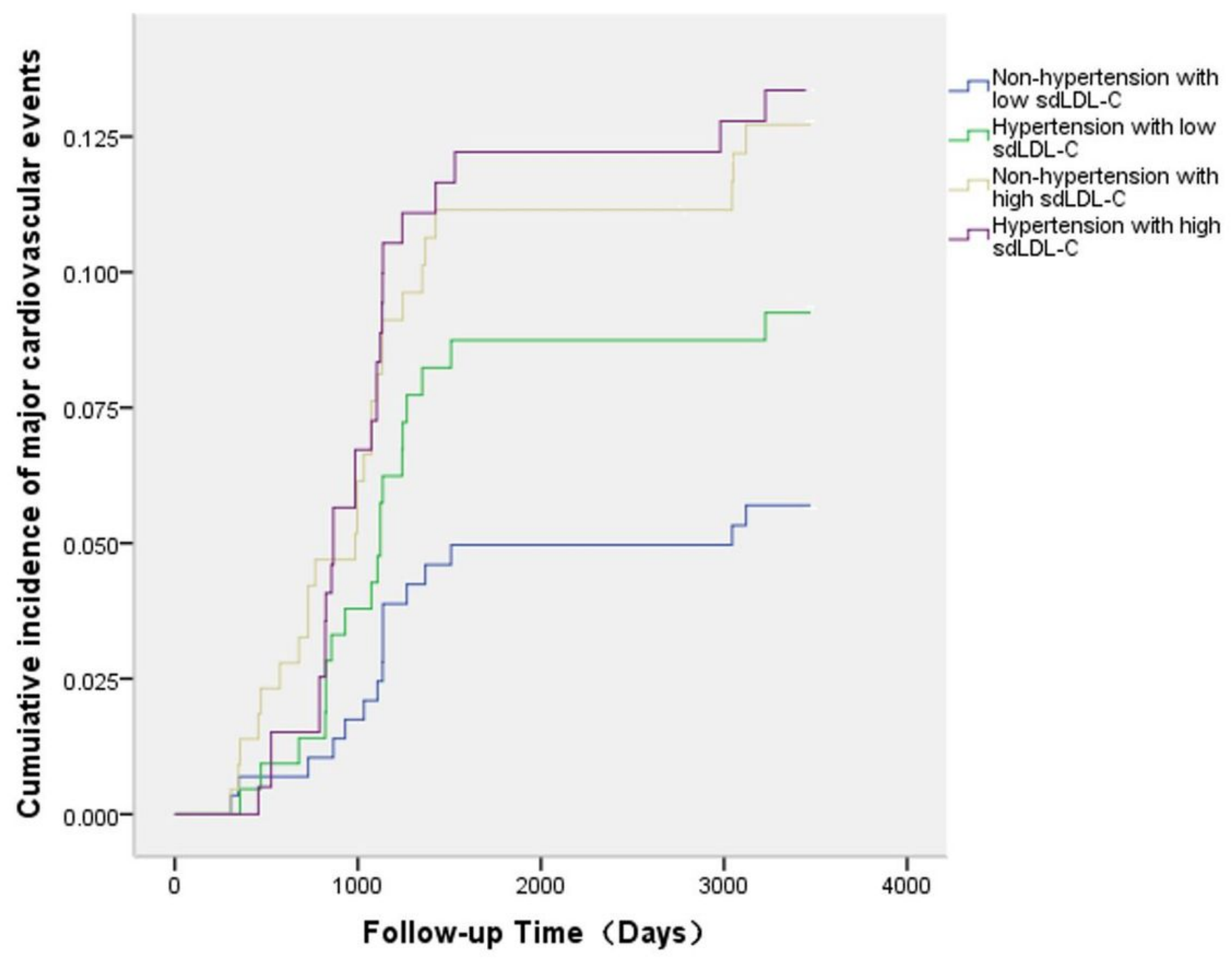

Figure 1

Risk for cardiovascular events by baseline sdLDL-C level. Kaplan-Meier survival curves indicating cumulative incidence of major adverse cardiovascular events across baseline sdLDL-C categories. Groups are indicated by colours; $p<0.001$ 


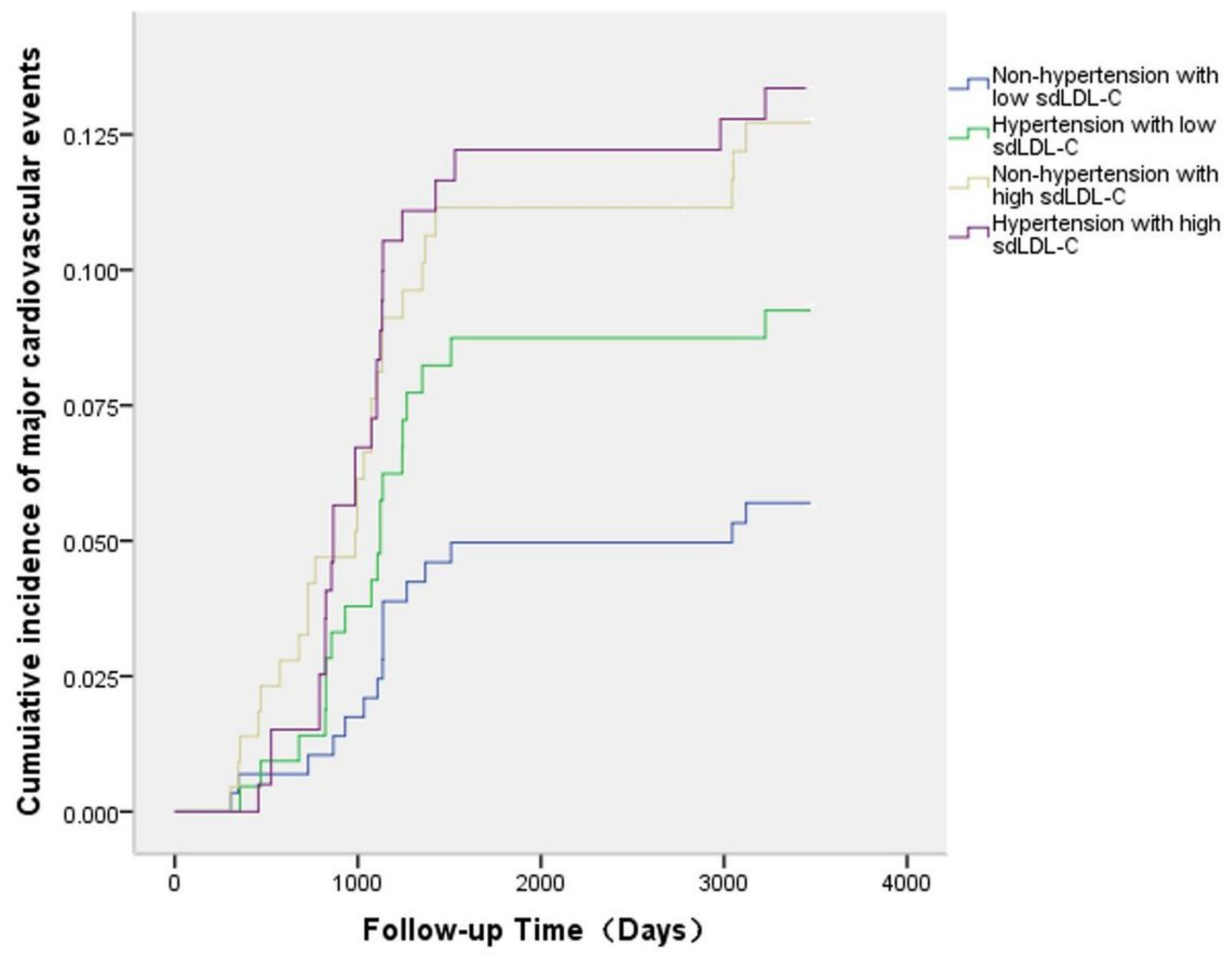

Figure 1

Risk for cardiovascular events by baseline sdLDL-C level. Kaplan-Meier survival curves indicating cumulative incidence of major adverse cardiovascular events across baseline sdLDL-C categories. Groups are indicated by colours; $p<0.001$ 